\title{
OBSERVATIONS OF HIGHLY-IONIZED INTERSTELLAR IRON
}

\author{
L. M. Hobbs
}

Yerkes Observatory, University of Chicago

$\underline{\text { ABSTRACT }}$

The spectra of 24 stars, including 5 at distances $d<200 \mathrm{pc}$, have been observed in the regions of the coronal [Fe X] $\lambda 6375$ and $[\mathrm{Fe}$ XIV] $\lambda 5303$ lines at detection limits near an equivalent width of $1 \mathrm{~mA}$ in the best cases. In general agreement with predictions based on a multi-phase model of the interstellar medium, no absorption which can be attributed to $\mathrm{Fe} X$ or $\mathrm{Fe} X I V$ ions in hot interstellar gas emitting the soft $x$-ray background is seen in any of these spectra, except for two. Toward $\lambda$ Cephei an absorption line near $\lambda 6375$ is measured with an equivalent width of $8.1 \pm 2 \mathrm{~mA}$, a width corresponding to $20 \pm 5 \mathrm{~km} \mathrm{~s}^{-1}$ or a temperature $\mathrm{T}<(0.5 \pm 0.25) \times 10^{6}{ }^{\circ} \mathrm{K}$, and, if it is caused by $\mathrm{Fe} \mathrm{X}$ ions, a radíal velocity of $-355 \mathrm{~km} \mathrm{~s}^{-1}$. On that hypothesis, the hot interstellar gas constitutes at least $63 \%$ of the column density of gas along this light path.

\section{INTRODUCTION}

The goal of the observations described here is to determine whether the physical properties, such as the space distribution, temperature, and kinematics, of the hot, diffuse, broadly distributed component of the interstellar gas can be observed directly, perhaps over kiloparsec path lengths, by a new method. Direct observations of this gas apparently have been possible hitherto only by virtue of its background emission at soft $x$-ray and extreme UV wavelengths (McCray and Snow 1979; Cowie et al. 1979). The new method consists of searching for the forbidden coronal [Fe X] $\lambda 6375$ and [Fe XIV] $\lambda 5303$ lines as extremely weak interstellar lines toward bright stars (Hobbs 1984; Hobbs and Albert 1984).

The method prospectively offers at least two advantages. (1) The ionization balance of $\mathrm{Fe}$ in steady-state collisional equilibrium in the temperature range $0.7 \leqslant \mathrm{~T}_{6} \leqslant 2$, where $\mathrm{T}_{6}$ is the temperature in units of $10^{6} \mathrm{~K}$, is generally favorable for Fe X (Shapiro and Moore 1976), in contrast to the cases of C IV, $\mathrm{N} V$, and $\mathrm{Si}$ II, which are present predominantly in the cooler surfaces of and transition regions around embedded cold clouds. The lower part of this temperature range is that expected for the hot gas, as judged from its soft $x$-ray emission (McCammon et a1. 1983). Non-equilibrium effects also could somewhat increase the ionization level of this gas, further enhancing its Fe $X$ fraction. (2) With ground-based telescopes of large aper- 
ture at which adequate amounts of observing time can be utilized for the search, very favorable detection limits at equivalent widths $W_{\lambda}<1 \mathrm{~mA}$ or $W_{\lambda} / \lambda<2 \times 10^{-7}$ are routinely achieved. This advantage is further enhanced by the moderate instrumental res olution required, owing to the thermal line broadening $\Delta \lambda \lesssim 0.5 \AA$ or $\Delta u \leqslant 25 \mathrm{~km} \mathrm{~s}^{-1}$ contributed by the hot gas. A third consideration is that a sensitive study of very weak 1ines near $\lambda \lambda 5303$, 6375 in suitable stellar spectra has not previously been reported.

The principal disadvantage of the method is that it relies upon detection of a single, broad, extremely weak line of each ion. The McKee-Ostriker (1977) model can be used to predict typical 1ine strengths $W_{\lambda}<0.1 \mathrm{~mA} ;$ for a 1 ine width $\Delta \lambda \lesssim 0.5$, the fractional absorption will not exceed $10^{-4}$, so that a detector capable of delivering a signal-to-noise ratio $\mathrm{S} / \mathrm{N} \approx 10^{-4}$ is required. Practical limits for existing detectors seem to be $\mathrm{S} / \mathrm{N} \lesssim 500$. Nonetheless, uncertainties about the ionization balance and the column-density fluctuations in the hot gas prompted this study of a potentially powerful diagnostic method for the hot interstellar gas.

RESULTS

The initial results obtained in the period 1979 to 1983 using the $2.7 \mathrm{~m}$ reflector of McDonald Observatory have been reported in two current papers (Hobbs 1984; Hobbs and Albert 1984). Observations made with the coude spectrograph and a dual-array Digicon detector were carried out for 32 stars at the [Fe $X]$ line and for 26 stars at the [Fe XIV] line, with 24 stars in common. Detector 1 imits near $W_{\lambda}=1 \mathrm{~mA}$, corresponding to $\mathrm{S} / \mathrm{N} \approx 400$, were achieved in the best cases. Catalogues of telluric and stellar lines, and of the diffuse interstellar bands at $\lambda \lambda 6376$ and 6379 , were established through observations of bright standard stars, to avoid erroneous identifications of such lines as [Fe X] or [Fe XIV] absorption. The discovery of about a dozen very weak, previously unreported telluric or stellar lines empirically attests to the unprecedented sensitivity, at these two spectral regions, of the search.

With two exceptions, the practical limit of $\mathrm{S} / \mathrm{N} \approx 400$ yielded negative results for interstellar Fe $X$ and $F e ~ X I V ~ a b-$ sorption, in general agreement with theoretical predictions. It should be emphasized that present telescopes are entirely adequate to achieve the required $S / N \approx 10^{4}$ in practical exposure times toward a suitable array of bright target stars, once future improvements in detector stability are achieved.

Uniquely toward $\lambda$ Cep and $v$ Cep, which are two highluminosity members of the Cepheus OB2 association, an absorption 
line which can be identified as [Fe X] $\lambda 6375$ is unmistakably present, with a strength $W_{\lambda}=8.1 \pm 2(3 \sigma) \mathrm{m} \AA$, a width $\Delta \mathrm{v}=20 \pm$ $5 \mathrm{~km} \mathrm{~s}^{-1}$ or $\mathrm{T}_{6} \leq 0.5 \pm 0.25$, and a radial velocity $\mathrm{v}_{\mathrm{r}}=-355$ $\mathrm{km} \mathrm{s}^{-1}$. The prēsence of the line in two stars separated by $\mathrm{d}>50 \mathrm{pc}$ demonstrates that the inferred hot gas is not locally confined near either star, owing, for example, to the impact of a stellar wind upon the ambient interstellar medium. The absorption apparently arises in an unusually dense, low-latitude concentration of hot interstellar gas. It may be possible to construct a simple, physically consistent model, which consists of a thin sheet of hot gas expanding outward from the plane and seen nearly edge on, which is consistent with other existing $x$-ray, UV, optical, and radio observational data for the region.

\section{REFERENCES}

Cowie, L. L., Jenkins, E. B., Songaila, A., and York, D. G. 1979p Ap. J. 232,467 .

Hobbs, L. M. 1984 , Ap. J. 280, 132 .

Hobbs, L. M., and Albert, C. E. 1984, Ap. J. 281, 639.

McCammon, D., Burrows, D. N., Slanders, W. T., and Kraushaar, W. L. 1983, Ap. J. 269, 107 .

McCray, R., and Snow, T. P. 1979, Ann. Rev. Astr. Ap. 17, 213.

McKee, C. F., and Ostriker, J. P. 1977, Ap. J. 218, 148.

Shapiro, P. R., and Moore, R. T. 1976, Ap. J. 207, 460. 